\title{
Situation à Three Mile Island après l'accident du 28 mars 1979
}

\section{Aspects de radioprotection}

\author{
C. CHEVALIER* \\ (Manuscrit reçu le 15 février 1983)
}

\begin{abstract}
RÉSUMÉ
Après un bref rappel de la chronologie de l'accident survenu sur le réacteur $n^{\circ} 2$ de Three Mile Island, l'auteur, qui a participé per sonnellement aux premières opérations de diagnostic sur le terrain, donne un apercu de la situation telle qu'elle se présentait fin 1981 . Les dégâts sont considérables et, malgré l'avancement des travaux de décontamination, les obstacles, essentiellement administratifs et financiers, n'ont pas permis, quatre ans après l'incident, d'avancer un pronostic sérieux sur la remise en état du réacteur endommagé.
\end{abstract}

\section{ABSTRACT}

After a brief flashback on the chronology of the accident that occured on TMI unit 2, the author, who took part in the first operations of diagnosis on the spot, gives an outlook of the situation at the end of 1981. The damages are considerable, and, in spite of the progression of decontamination works, the obstacles, mainly administrative and financial, do not allow, four years after the event, to give a serious forecast on the repairing of the damaged reactor.

\section{INTRODUCTION}

L'accident survenu sur la tranche 2 le 28 mars 1979 à 4 h du matin a projeté la centrale américaine de Three Mile Island à l'avant-scène de l'actualité. Une vanne de décharge restée bloquée en position ouverte, la non reconnaissance par les exploitants de cette situation à cause doune indication ambiguë en salle de commande ont été à l'origine de ce qu'on a pris l'habitude d'appeler "le plus grave accident du nucléaire civil". Cet événement a valu à une région paisible de Pennsylvanie jusqu'alors inconnue, de faire la une de tous les journaux du monde pendant plusieurs jours. Contrairement à ce qu'aurait pu laisser croire la multitude de gros titres et articles catastrophes parus dans la presse à l'époque, cet accident n'a finalement pas eu d'impact significatif sur l'environnement.

Cependant, les dégâts qui en ont résulté pour l'installation elle-même sont considérables et, à la fin de l'année 1981, soit près de 3 ans après

* EdF, centrale de Flammanville, BP 4, 50340 Les Pieux. 
Paccident, la tâche à accomplir pour décontaminer TMI 2 restait gigantesque, l'exploitant se trouvant confronté à de difficiles problèmes d'ordres financier, législatif et technique.

\section{LE CONTEXTE}

\subsection{L'exploitant}

Le propriétaire de TMI, General Public Utilities (GPU) est une société de type holding qui possède trois filiales d'exploitation en Pennsylvanie et dans le New Jersey (Metropolitan Edison, Jersey Central Power and Light, Pennsylvania Electric).

L'ensemble du groupe emploie environ 11500 personnes, alimente 1,5 million d'abonnés et occupe le $1^{\circ}$ rang des quelque 200 compagnies d'électricité américaines cotées en bourse.

Si GPU dispose d'une capacité totale de production d'environ $12000 \mathrm{MWe}$ dont P'essentiel est constitué par des centrales au charbon ou au fuel, le nucléaire, lui, représentait $2300 \mathrm{MWe}$ installés - 1 réacteur BWR (Oyster Creek, $620 \mathrm{MWe}$, mis en service en 1969) et 2 PWR (TMI 1, $800 \mathrm{MWe}$, mis en service en septembre 1974 et TMI 2, 900 MWe, mis en service le 30 décembre 1979 soit 3 mois avant l'accident).

Metropolitan Edison était l'exploitant agréé par la NRC (1) pour les 2 tranches de TMI et Jersey Central celui d'Oyster Creek. Après l'accident de TMI 2, GPU a décidé de regrouper ses activités dans le nucléaire et a créé une nouvelle filiale, GPU Nuclear, qui a reçu en 1981, l'agrément de la NRC pour exploiter l'ensemble des tranches nucléaires du groupe.

\subsection{Etat de la tranche 1 - Conséquences financières}

En mars 1979, la tranche 1 se trouvait en fin d'arrêt programmé pour rechargement ; elle n'a pas été touchée par les événements qui se sont produits le 28 sur la tranche 2.

On se souvient qu'après l'accident, la NRC a ordonné l'arrêt de toutes les tranches de type $B$ et $W(2)$ (identiques à celles de TMI) puis a autorisé assez rapidement leur remise en service, sauf toutefois celle de TMI 1, considérant que cette tranche constituait un cas particulier notamment à cause des intéractions entre les 2 unités au niveau du BAN (3) et aux incidences éventuelles des activités de décontamination de TMI 2 sur TMI 1.

L'enquête publique ("hearings") ordonnée par la NRC devait durer normalement un an, mais elle n'a, en fait, commencé qu'en octobre 1980 (soit un an et demi après l'accident) et s'est terminée en septembre 1981. La NRC semblait sur le point de donner l'autorisation de remise en service mais l'exploitant a découvert, en fin d'année 1981, pendant les essais préliminaires, de nombreuses fuites sur les générateurs de vapeur (plusieurs centaines de tubes) dues, sans doute, à un problème de conservation.

(1) Nuclear regulatory commission.

(2) Babcock et Wilcox.

(3) Bâtiment des auxiliaires nucléaires. 
La réparation risque d'être assez longue. Comme, par ailleurs, les opposants au nucléaire en général et à TMI en particulier n'ont pas désarmé et ont même entrepris de nouvelles actions en justice, il est à craindre que la remise en service de TMI 1 ne soit pas encore pour 1983. S'il existe maintenant un problème technique, au début, le maintien à l'arrêt de TMI 1 était dû à un problème strictement réglementaire. II est certain que l'indisponibilité prolongée de TMI 1, ajoutée bien évidemment à celle de TMI 2 , représente un manque à gagner important pour GPU, ce qui n'est pas fait pour arranger une situation financière bien compromise depuis trois ans.

\subsection{Financement des travaux de TMI 2}

Une étude déjà ancienne de la NRC (1980) a estimé le coût de la décontamination à 1 milliard de $\$$. Des études plus récentes laissent à penser que ce chiffre sera largement dépassé. Dans l'état actuel des choses, il est d'ailleurs bien difficile d'estimer aussi bien la durée que le coût de l'opération. De toute façon, le montant de la facture est tel que GPU, de par sa taille, n'est pas en mesure d'en assurer seul le réglement. II faut d'ailleurs ajouter aux opérations de décontamination le manque à gagner en raison de l'indisponibilité des 2 tranches, estimé à 500000 dollars par jour et par réacteur.

Dans le courant de 1981 de nombreuses personnalités politiques se sont préoccupées du problème du financement de TMI 2 en particulier des élus de la Pennsylvanie. Parmi les différentes propositions, celle du Gouverneur de l'Etat, Richard Thornburgh semblait le plus retenir l'attention. Ce projet proposait, en fait, un partage par moitié entre des ressources locales (GPU et les Etats de Pennsylvanie et du New Jersey) et des ressources nationales (Etat fédéral et une association des compagnies d'électricité américaines ayant des activités dans le nucléaire).

Depuis le début de l'année 1982, la situation semble avoir rapidement évolué. En particulier, la NRC aurait chargé le DOE (4) de prendre à son compte toutes les opérations liées au déchargement du combustible. Par ailleurs, les américains ont pris contact avec la plupart des pays occidentaux pour leur demander une participation à la fois financière et technique aux opérations de décontamination de TMI 2.

\section{L'ACCIDENT ET SES CONSÉQUENCES POUR LE CCEUR DU RÉACTEUR}

\subsection{Rappel de la chronologie de l'accident}

L'accident de la tranche 2 s'est produit le 22 mars 1979 à 4 h du matin soit trois mois seulement après la mise en service industrielle. Le réacteur fonctionnait à $97 \%$ de sa puissance nominale lorsqu'un problème sur le réseau d'air comprimé a entraîné la fermeture des vannes d'aspiration des pompes alimentaires, puis le déclenchement de ces pompes et des pompes d'extraction. La perte simultanée de l'eau alimentaire sur les deux

(4) Department of energy. 
générateurs de vapeur a provoqué le déclenchement de la turbine et le démarrage des trois pompes d'alimentation de secours. Cet instant est défini comme le temps $t=0$ dans la chronologie de l'accident. La vanne de décharge du pressuriseur s'est normalement ouverte à $t=3 \mathrm{~s}$ sur signal de haute pression (158 bars) et l'arrêt d'urgence du réacteur s'est produit à $\mathrm{t}=8 \mathrm{~s}$ sur signal de très haute pression (164 bars). Une indication ambiguë en salle de commande laissa croire aux opérateurs que la vanne de décharge s'était normalement refermée à $t=13 \mathrm{~s}$ (154 bars) alors qu'elle était, en fait, restée bloquée ouverte. La remontée du niveau du pressuriseur à partir de $\mathrm{t}=1 \mathrm{~min}$ conforta les opérateurs dans leur première analyse (vanne fermée), ce qui les amena à passer en contrôle manuel l'injection de sécurité haute pression ( $t=3 \min 14 \mathrm{~s}$ ) alors que deux des trois pompes étaient en service ( $A$ et $C$ ) puis à arrêter la pompe $C$ et laminer les vannes au refoulement de la pompe $A(t=4 \min 38 \mathrm{~s})$.

Côté secondaire, les pompes d'alimentation de secours des générateurs de vapeur (ASG) ont atteint leur pression de refoulement ( $t=14 \mathrm{~s}$ ) et les vannes automatiques d'alimentation se sont normalement ouvertes sur signal de niveau bas GV $(t=30 \mathrm{~s})$. Mais le système ASG s'est révélé inopérant car des vannes manuelles d'isolation avaient été laissées fermées à la suite d'essais réalisés sur les pompes quelques jours avant l'accident. Les générateurs de vapeur s'assèchèrent en $2 \mathrm{~min}$ environ. L'ASG fut finalement rétabli à $\mathrm{t}=8 \mathrm{~min}$ après intervention d'un exploitant sur les vannes d'isolation.

Ce problème sur l'alimentation de secours n'a pas eu de conséquence directe sur l'accident puisque la vanne de décharge du pressuriseur s'est bloquée à $t=13 \mathrm{~s}$ et que le niveau bas du GV n'a été atteint qu'à $t=30 \mathrm{~s}$. Cependant, il est difficile d'estimer l'impact psychologique que ce fait a pu avoir sur l'exploitant qui a eu, sans aucun doute, son attention détournée vers le circuit secondaire alors que le vrai problème se trouvait du côté primaire.

Ce n'est qu'à $t=2 \mathrm{~h} 22 \mathrm{~min}$ que les opérateurs ont réalisé enfin leur erreur et ont isolé la ligne de décharge du pressuriseur. Pendant toute cette période, l'injection de sécurité n'a été mise en service que de façon intermittente, le circuit primaire s'est lentement vidé et le cœur a été en partie dénoyé.

Pendant les heures suivantes les opérateurs ont tenté de rétablir la circulation forcée dans le cœur en redémarrant les pompes primaires, la circulation naturelle ne pouvant s'établir à cause de la présence d'une bulle de vapeur et d'hydrogène dans la partie haute de la cuve.

Après plusieurs essais infructueux, les pompes primaires ont pu être remises en service vers $\mathrm{t}=4 \mathrm{~h}$ et à partir de $\mathrm{t}=10 \mathrm{~h}$ les températures des branches chaudes et froides ont commencé à converger, indiquant qu'une circulation était rétablie dans le cœur. On peut considérer que la situation était redevenue à peu près stable dans le réacteur vers $t=22 \mathrm{~h}$.

\subsection{Estimation des dégâts subis par le cœur}

Le cœur a subi d'importants dommages; de nombreuses indications permettent de l'affirmer: 
- on a mesuré des activités très importantes dans les effluents ;

- on sait qu'une combustion d'hydrogène s'est produite à $\mathrm{t}=10 \mathrm{~h}$ dans le bâtiment réacteur, elle a provoqué un pic de pression d'environ 2 bars et le déclenchement de l'aspersion. La production de grandes quantités de ce gaz par réaction entre le zircaloy et l'eau suppose que les gaines ont atteint des températures très élevées ;

- à $t=222 \min$ (après renoyage) on a observé une remontée rapide de réactivité, de la pression primaire (bien que la vanne de décharge du pressuriseur soit ouverte à ce moment-là) et des températures des branches froides (les températures des branches chaudes étaient déjà hors d'échelle, donc supérieures à $325^{\circ} \mathrm{C}$ ). Ce phénomène est attribué à un effondrement partiel du cœur et une redensification des débris. Après l'accident, des études de pertes de charge du cœur effectuées séparément par Babcock et Batelle confirment qu'une large partie du cœur est obstruée.

Jusqu'à la fin de 1981, aucune observation directe du cœur n'avait pu être encore effectuée. Un examen à l'aide d'une caméra miniature qui serait introduite par l'orifice d'un mécanisme de grappe était prévu pour la fin du premier semestre 1982.

Néanmoins, de nombreuses études ont été entreprises pour tenter de préciser les dégâts subis par le cœur. Trois approches ont été utilisées :

- la simulation par des codes de calcul du transitoire thermohydraulique ;

- l'évaluation de la quantité d'hydrogène produite par la réaction zircaloyeau ;

- l'étude du relâchement des produits de fission.

A partir de ces différents calculs, il a été possible de définir trois niveaux de dégâts pour le cœur : un minimum (hypothèses basses), un maximum (hypothèses pessimistes) et un état moyen dit cœur de référence. Le tableau I résume les résultats obtenus.

Les simulations par les codes de calcul ont été effectuées par plusieurs organismes (NRC, Batelle Colombus, Westinghouse, laboratoires de Los Alamos). Les auteurs de ces études sont tous d'accord pour estimer que le maximum de dégâts sur le cœur s'est produit entre $t=100 \mathrm{~min}$ (arrêt des pompes primaires) et $t=210 \mathrm{~min}$ (fin du renoyage). La première rupture de gaine serait apparue vers $t=140 \mathrm{~min}$.

Aucune quantité significative de combustible n'a atteint la température de fusion de l'oxyde d'uranium $\left(2800^{\circ} \mathrm{C}\right)$. Ce point est confirmé par l'étude du relâchement des produits de fission (faible teneur en tellure et ruthénium).

Les assemblages périphériques sont vraisemblablement tous ruptés mais ils ont dû conserver leur intégrité géométrique. La partie centrale du cœur s'est effondrée. On estime qu'un matelas de débris a dû se former dans le plan médian du cœur et qu'il s'étend sans doute jusqu'à la limite des assemblages périphériques. 
C. CHEVALIER

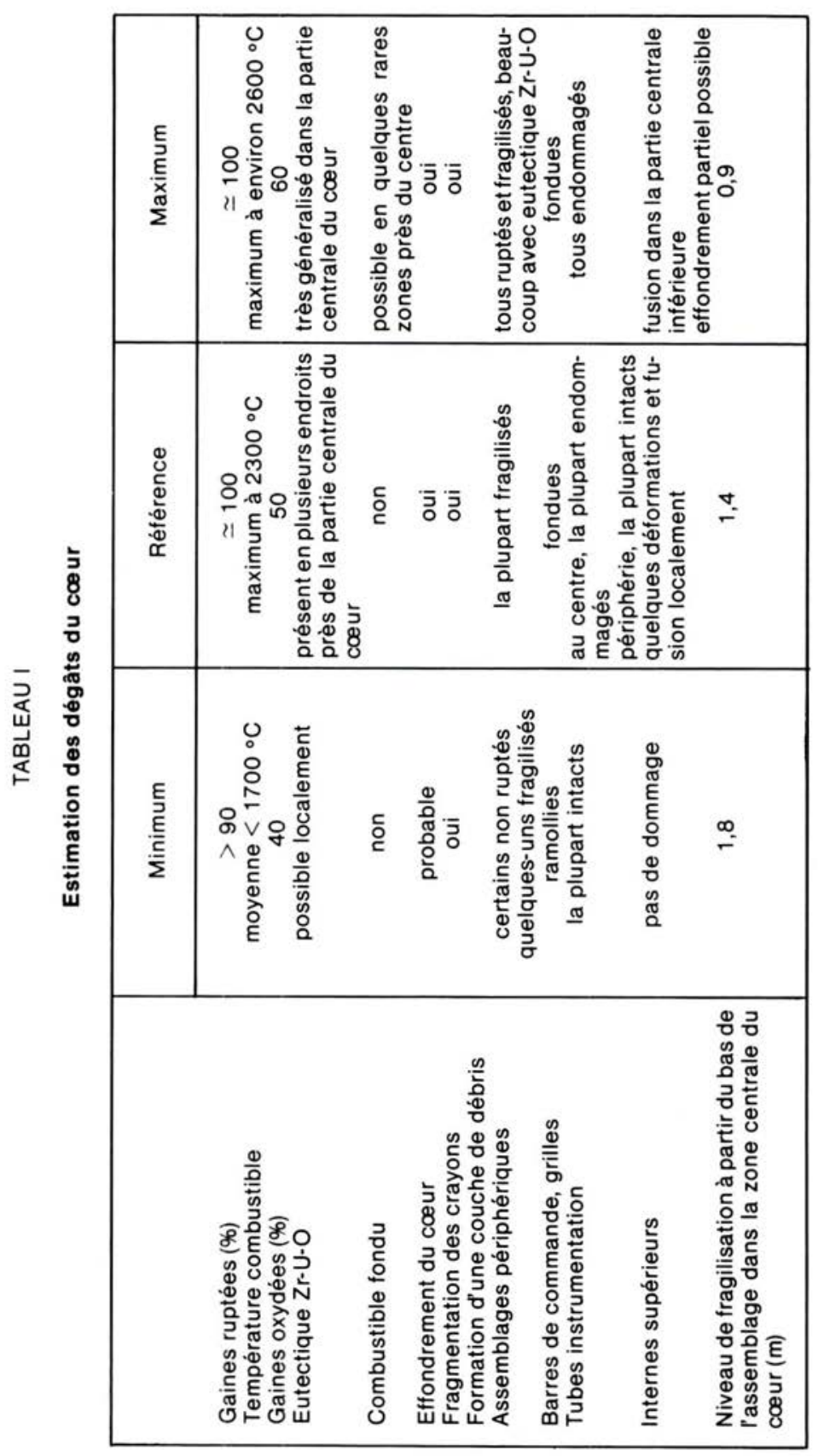




\section{PROBleme des EFFLUeNTS ET DES DEChETS SOLIDES}

\subsection{Effluents radioactifs gazeux}

Au moment de l'accident, d'importantes quantités de gaz de fission et d'iode se sont échappées du circuit primaire. En dehors de quelques rejets incontrôlés extrêmement limités, la quasi-totalité de ces gaz est restée confinée dans l'enceinte du bâtiment réacteur.

Avant de pouvoir pénétrer à l'intérieur pour effectuer les premières investigations, il était nécessaire d'évacuer ces gaz. La NRC s'est livrée à de nombreuses études et a envisagé plusieurs solutions (purge à l'atmosphère, absorption sélective, absorption sur charbon, stockage sous forme de gaz comprimés, séparation cryogénique). Etant donné l'impact très faible sur l'environnement qu'avait en fait le rejet direct à l'atmosphère, c'est cette solution qui a été finalement retenue.

Ces études ont été longues et l'exploitant a eu quelques difficultés pour obtenir toutes les autorisations nécessaires. Lorsque les opérations ont pu commencer le 28 juin 1980, soit 15 mois, jour pour jour, après l'accident, les radioéléments à vie courte comme l'iode 131 (période 8 j) ou le xénon 133 (période $5,3 \mathrm{j}$ ) avaient totalement disparu. Seul, restait le krypton 85 (période 10,6 ans) dont l'activité totale avait été estimée à $57000 \mathrm{Ci}\left(2.10^{15} \mathrm{~Bq}\right)$.

\subsection{Traitement des effluents liquides}

L'accident du 28 mars a produit de grandes quantités d'effluents liquides qui ont été classés en deux catégories selon que leur activité volumique était inférieure ou supérieure à $100 \mathrm{Ci} / \mathrm{m}^{3}\left(3,7.10^{12} \mathrm{~Bq} / \mathrm{m}^{3}\right)$.

Pour traiter de tels volumes et de telles activités, il a fallu construire deux unités spéciales: l'une, EPICOR II, pour les effluents les moins actifs $\left(<100 \mathrm{Ci} / \mathrm{m}^{3}-3,7.10^{12} \mathrm{~Bq} / \mathrm{m}^{3}\right)$, l'autre, SDS (5), pour les effluents les plus actifs $\left(>100 \mathrm{Ci} / \mathrm{m}^{3}-3,7.10^{12} \mathrm{~Bq} / \mathrm{m}^{3}\right)$.

L'installation EPICOR II comprend une cuve de stockage amont, trois pots de résines organiques en série, des pompes de transfert, une cuve tampon aval. Elle a été construite dans un local prévu pour recevoir l'atelier de décontamination du site. Entre octobre 1979 et août 1980, cette unité a traité environ 500000 gallons $\left(1900 \mathrm{~m}^{3}\right)$ d'eau d'activité en général inférieure à $30 \mathrm{Ci} / \mathrm{m}^{3}\left(1,1.10^{12} \mathrm{~Bq} / \mathrm{m}^{3}\right)$, elle a utilisé 69 pots de résines sur lesquels ont été fixés près de $55000 \mathrm{Ci}\left(2.10^{15} \mathrm{~Bq}\right)$.

SDS a été conçu pour traiter l'eau fortement active $\left(>100 \mathrm{Ci} / \mathrm{m}^{3-}\right.$ $3,7.10^{12} \mathrm{~Bq} / \mathrm{m}^{3}$ ) qui se trouve d'une part dans le bas du bâtiment réacteur $\left(2600 \mathrm{~m}^{3}\right)$ et d'autre part dans le circuit primaire $\left(340 \mathrm{~m}^{3}\right)$. Le réacteur ayant été accidenté trois mois seulement après sa mise en service, la piscine de désactivation n'avait jamais servi ; il a donc été décidé de construire au fond de cette piscine une chaîne de déminéralisation conçue pour fonctionner sous 6 à $7 \mathrm{~m}$ d'eau qui servira d'écran biologique. Le système peut être divisé en 5 grands modules :

(5) Submerged demineralizer system. 
- filtration ;

- déminéralisation sur zéolite ;

- épuration continue de l'eau de la piscine ;

- circuit d'échantillonnage ;

- système de traitement des gaz.

II a fallu de nombreux mois pour concevoir et construire ce système puis obtenir les autorisations de mise en service. Le traitement de l'eau du bâtiment réacteur a effectivement commencé en septembre 1981. Fin décembre 81 , la moitié de l'eau environ avait été traitée. L'opération s'est terminée en mai 82. SDS a fonctionné de façon tout à fait satisfaisante avec de très bons facteurs de décontamination en césium $\left(10^{5}\right)$ et en strontium (500).

Le traitement de l'eau est ensuite complété par passage sur EPICOR II. L'utilisation successive de ces deux unités permet une décontamination tout à fait remarquable des effluents de forte activité du bâtiment réacteur. La contamination résiduelle (hors tritium) est principalement due au strontium 90 (environ $10^{-5} \mathrm{Ci} / \mathrm{m}^{3}-3,7 \mathrm{MBq} / \mathrm{m}^{3}$ ) et au césium (environ $5.10^{-6}$ $\left.\mathrm{Ci} / \mathrm{m}^{3}-0,2 \mathrm{MBq} / \mathrm{m}^{3}\right)$.

\subsection{Déchets solides}

Pour l'instant, la principale source de déchets radioactifs solides de forte activité vient des pots de résines usées de SDS et EPICOR II. Les 22 résines usées les moins actives résultant du fonctionnement d'EPICOR II ont été évacuées courant 1981 vers le centre de stockage fédéral de Hanford (Etat de Washington). Les autres sont provisoirement stockées sur le site dans des silos en béton construits dans ce but après l'accident. Les résines SDS, qui peuvent être chargées jusqu'à $60000 \mathrm{Ci}\left(2,2.10^{15} \mathrm{~Bq}\right)$, sont pour l'instant stochées sous eau dans la piscine de désactivation. Le DOE a entrepris une étude sur la vitrification des zéolites de SDS.

De toute façon, la NRC a clairement fait savoir que le site de TMI ne devait pas devenir un centre de stockage et que, par conséquent, des solutions devraient être trouvées pour évacuer tous les déchets radioactifs solides résultants des activités de décontamination de la tranche 2.

\section{LES OPÉRATIONS DE NETTOYAGE}

\subsection{Les différentes phases}

Après l'accident, GPU a demandé à la société Bechtel de prendre en charge les études et la réalisation de l'ensemble des opérations. Le programme de nettoyage a été divisé en sept phases principales :

- traitement de l'eau radioactive contenue dans le bâtiment des auxiliaires nucléaires (BAN) ;

- décontamination de ce bâtiment ;

- élimination des gaz radioactifs contenus dans l'enceinte de confinement ;

- traitement de l'eau fortement contaminée qui se trouve dans l'enceinte et le circuit primaire ; 
- décontamination grossière des sols et parois du bâtiment réacteur (BR) ;

- démontage du cœur ;

- décontamination finale poussée.

Nous avons vu, dans le chapitre précédent, que l'eau du BAN a été traitée entre octobre 1979 et août 1980 par EPICOR II, et celle du BR entre la fin de 1981 et mai 1982 par SDS, enfin que les gaz radioactifs contenus dans l'enceinte ont été évacués dans l'atmosphère en juin 1980.

Dans les mois qui ont suivi l'accident, un large effort de décontamination a été engagé dans le BAN. Ce bâtiment, qui comprend trois niveaux, était décontaminé à environ $80 \%$ à la mi 82 et de nombreuses zones sont désormais accessibles sans protection particulière. Un travail important reste à faire au niveau inférieur, mais il ne présente pas un caractère d'urgence. On y trouve des zones qui avaient été très fortement contaminées, en particulier des déminéraliseurs et un évaporateur pour le traitement des effluents liquides, les pompes de charge du RCV (6), les cuves de stockage de résines usées, des locaux de vannes diverses, le puisard général du bâtiment.

Ces opérations dureront sans doute encore plusieurs années.

\subsection{Les opérations réalisées dans le bâtiment réacteur}

Après la purge du krypton 85 (juin 1980), la première pénétration dans le bâtiment réacteur a eu lieu le 23 juillet 1980 . L'équipe était constituée par deux personnes (choisies dans une liste de volontaires) qui sont restées $20 \mathrm{~min}$ dans l'enceinte et ont reçu une dose de 200 mrem environ (2 mSv).

Pendant un peu plus d'un an, ces entrées se sont poursuivies à la cadence moyenne d'une par mois avec comme principaux objectifs :

- établir une cartographie aussi précise que possible des débits de dose et des niveaux de contamination ;

- prendre des photos ;

- mettre en place un système de caméras vidéo et quelques appareils de radioprotection pour remplacer ceux qui étaient défaillants ;

- effectuer quelques essais de décontamination du sol par eau basse ou haute pression, ou par enduit préalable ;

- mettre en place la pompe flottante et les tuyauteries pour transférer l'eau du bâtiment réacteur vers SDS.

Vers la fin de l'année 1981, la cadence des entrées s'est accélérée pour atteindre en moyenne une par semaine.

Deux objectifs principaux sont visés :

- la remise en état du pont roulant principal :

- la décontamination grossière des sols au niveau de l'entrée (élévation $305 \mathrm{ft}$, plan médian du cœur) et au niveau supérieur (élévation $347 \mathrm{ft}$, plancher piscine).

(6) Système de contrôle volumétrique et chimique du réacteur. 


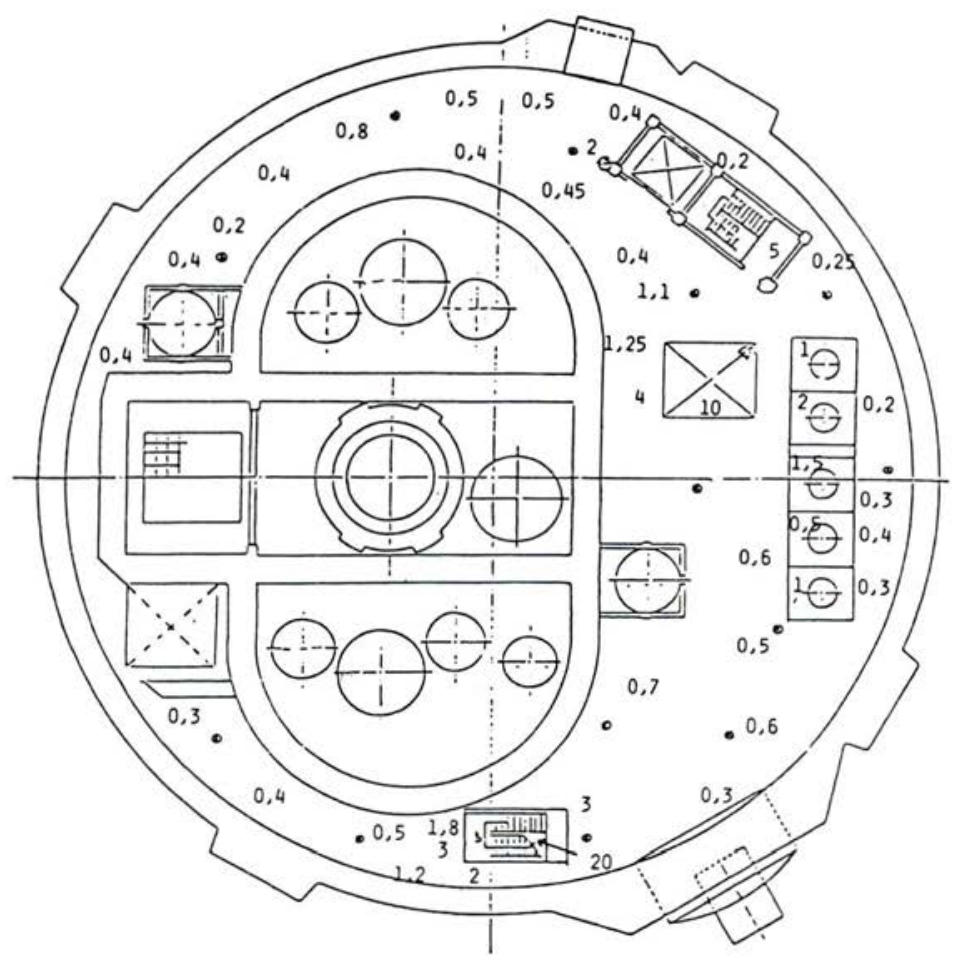

Fig.1. - Débit de dose $\gamma$ en rem/h à $1 \mathrm{~m}$ du sol - Niveau $305 \mathrm{ft}$.

L'ambiance générale est d'environ 400 à $500 \mathrm{mrem} / \mathrm{h}$ (4 à $5 \mathrm{mSv} / \mathrm{h}$ ) au niveau $305 \mathrm{ft}$ et de 200 à $300 \mathrm{mrem} / \mathrm{h}(2 \mathrm{à} 3 \mathrm{mSv} / \mathrm{h})$ au niveau $347 \mathrm{ft}$ (fig. 1 et 2). On observe, bien sûr, un certain nombre de points chauds : les siphons de sol, les traversées dans le plancher en béton. Une part importante du débit de dose vient, sans doute, du rayonnement en provenance de l'eau très active qui se trouvait toujours au fond du bâtiment réacteur à l'époque où ont été effectuées ces mesures (ce qui explique les points chauds au niveau des traversées du plancher).

Le tableau II résume les doses cumulées pour les 14 premières entrées, soit pendant un an. La dose la plus forte prise par une seule personne a été de 820 mrem $(8,2 \mathrm{mSv})$. Les doses les plus importantes ont été prises par les trois agents qui ont participé à l'entrée $n^{\circ} 8$, à savoir 570 , 770 et 820 mrem $(5,7-7,7$ et $8,2 \mathrm{mSv})$ pour des temps de séjour de 40 , 40 et $25 \mathrm{~min}$. II s'agissait d'opérations effectuées vers le fond du bâtiment réacteur, à proximité de l'eau, en particulier la mise en place d'une pompe flottante et des tuyauteries nécessaires pour alimenter SDS. 
SITUATION A THREE MILE ISLAND APRES L'ACCIDENT DU 28 MARS 1979

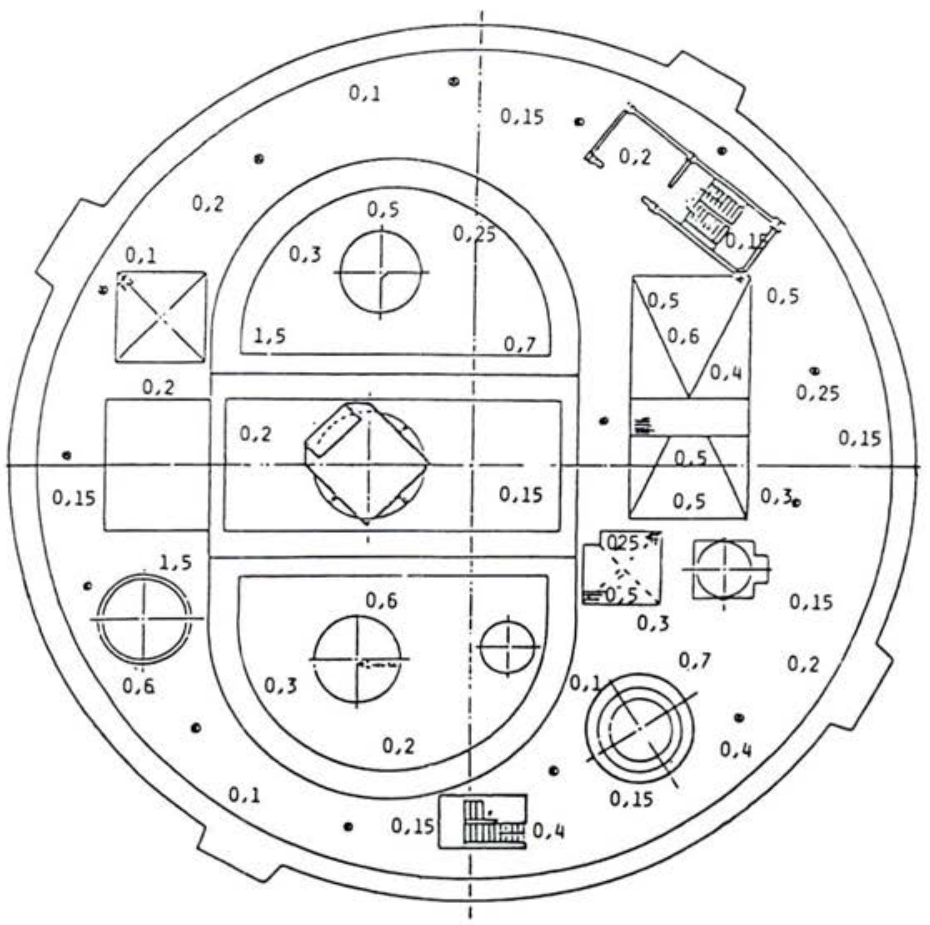

Fig. 2. - Débit de dose $\gamma$ en rem/h à $1 \mathrm{~m}$ du sol - Niveau $347 \mathrm{ft}$.

TABLEAU ॥

Dosimétrie pour les 14 premières entrées dans le bâtiment réacteur

\begin{tabular}{|c|c|c|c|c|}
\hline No entrée & Date & $\begin{array}{c}\text { Nombre de } \\
\text { personnes }\end{array}$ & $\begin{array}{c}\text { Durée totale } \\
\text { Hommes } \times \text { heures }\end{array}$ & $\begin{array}{c}\text { Dose totale } \\
\text { Hommes } \times \text { rems }\end{array}$ \\
\hline 1 & $23-7-1980$ & 3 & 2,30 & 0,45 \\
\hline 2 & $15-8-1980$ & 4 & 2,96 & 1,07 \\
\hline 3 & $16-10-1980$ & 5 & 5,22 & 2,28 \\
\hline 4 & $13-11-1980$ & 12 & 13,45 & 4,11 \\
\hline 5 & $11-12-1980$ & 14 & 18,92 & 5,90 \\
\hline 6 & 3 et $5-2-1981$ & 32 & 45,44 & 11,99 \\
\hline 7 & $17,19,20-3-1981$ & 13 & 19,95 & 4,915 \\
\hline 8 & $8-4-1981$ & 3 & 1,78 & 2,16 \\
\hline 9 & $30-4-1981$ & 8 & 5,08 & 3,04 \\
\hline 10 & $14-5-1981$ & 23 & 20,85 & 6,50 \\
\hline 11 & $28-5-1981$ & 16 & 25,75 & 4,49 \\
\hline 12 & $25-6-1981$ & 6 & - & 2,12 \\
\hline 13 & $1-7-1981$ & 4 & - & 1,20 \\
\hline 14 & $23-7-1981$ & 9 & - & 4,00 \\
\hline
\end{tabular}


En moyenne, les doses varient de 200 à 500 mrem (2 à 5 mSv) pour des temps de séjour allant de $30 \mathrm{~min}$ à $2 \mathrm{~h}$.

\section{CONCLUSION}

Les exploitants de TMI restent confrontés à de difficiles problèmes d'ordre financier, technique, législatif. Près de 3 ans après l'accident, les travaux de nettoyage n'étaient encore que dans une phase préliminaire. Aucune opération d'envergure n'avait été réalisée à l'intérieur du bâtiment réacteur et l'essentiel des actions engagées en sont au stade des études.

En dehors du traitement des effluents, GPU avait, fin 1981, deux objectifs principaux au niveau du bâtiment réacteur: la décontamination grossière des sols par de l'eau sous pression, la remise en état du pont roulant principal.

Cette dernière opération était rendue difficile par des problèmes d'accès, aussi bien au niveau du pont lui-même que, d'une façon générale, dans le bâtiment réacteur. L'exploitant ne disposait encore que du sas d'entrée du personnel, par le bâtiment des auxiliaires nucléaires, sas de dimension réduite, prévu pour quelques personnes et ne permettant pas de faire entrer des matériels volumineux ou lourds (toutes les manutentions devant se faire à la main). L'ouverture dans l'enceinte permettant normalement le transfert des gros composants et improprement appelée sas matériel, ne pouvait être utilisée, car ce n'est justement pas un sas et qu'elle mettait en communication directe l'intérieur du bâtiment réacteur fortement contaminé avec l'environnement.

La société Bechtel a donc entrepris pour le compte de GPU l'étude d'un local qui serait construit contre le bâtiment réacteur, permettrait de créer effectivement un sas et contiendrait différents aménagements indispensables pour la suite des opérations (atelier chaud, atelier de décontamination, vestiaires actifs). Ce projet (Containment service building) se heurtait, fin 1981, à quelques problèmes à la fois d'ordre financier è législatif. La NRC semble, en effet, considérer ce bâtiment comme une extension de l'enceinte de confinement ; il devrait donc satisfaire aux mêmes critères (confinement, tenue au séisme, etc.) et nécessiter les mêmes démarches administratives, sans doute très longues. Ce projet a donc pris beaucoup de retard.

Pourtant, aucune opération importante ne pourra avoir lieu sans ce bâtiment. On peut ne citer qu'un seul exemple : la requalification du pont roulant qui conditionne la suite des travaux (ouverture de la cuve, etc.) 2 tests en statique sont prévus : un à $50 \mathrm{t}$ en juillet 1983 et un deuxième à $200 \mathrm{t}$ en octobre 1983. On voit mal comment on pourrait introduire de telles charges à l'intérieur de l'enceinte dans les conditions actuelles.

Si les délais ci-dessus peuvent être tenus, il serait possible d'ouvrir la cuve à partir de 1984 puis d'entreprendre la dépose des internes et le déchargement du combustible (sans doute jusqu'en 1985). II est toutefois difficile de savoir aujourd'hui combien de temps sera nécessaire pour obtenir toutes les autorisations puis pour mener à bien cette difficile opération. II restera ensuite à terminer la décontamination de tous les circuits et du bâtiment réacteur. 
Etant donnée la durée de ces travaux, des dégradations importantes vont se produire un peu partout sur les installations d'autant plus que, par manque de moyens financiers, très peu d'opérations de maintenance sont effectuées.

GPU ne se prononce pas, pour l'instant, sur une éventuelle tentative de remise en service de TMI 2. Cette hypothèse semble très peu probable.

\section{ADDITIF}

L'action en justice intentée en novembre 1982 par GPU, propriétaire de TMI, contre Babcock et Wilcox, par laquelle l'exploitant réclamait au constructeur la somme de 4 milliards de dollars de dommages et intérêts, s'est finalement terminée par un compromis entre les deux compagnies, au terme duquel B et W paiera 37 millions de dollars à GPU sous forme de rabais sur les "services nucléaires" au cours des dix prochaines années.

Par contre, GPU poursuit son action contre la NRC, l'accusant de ne pas l'avoir correctement informé des risques sur le plan de la sûreté d'accidents semblables.

En ce qui concerne la tranche jumelle de TMI $n^{\circ} 1$, les obstacles à son redémarrage se lèvent progressivement :

- côté technique, les réparations nécessaires ont été effectuées, notamment sur le générateur de vapeur ;

- côté administratif, la Cour Suprême a statué le 19 avril 1983 que la NRC n'avait pas à tenir compte du "stress psychologique" des populations voisines pour prendre sa décision de redémarrer ou non la tranche 1.

Par ailleurs, sur la tranche accidentée, la requalification du pont était prévue durant le premier semestre 1983, préalablement à l'ouverture de la cuve (qui devrait intervenir mi-83).

\section{ANNEXE}

\section{TMI 2 : EQUIPEMENT NECESSAIRE POUR RENTRER DANS L'ENCEINTE DE CONFINEMENT}

On trouvera ci-dessous la liste des équipements nécessaires pour pénétrer à Pintérieur du bâtiment réacteur. On peut considérer qu'il s'agissait de l'équipement standard jusqu'à la fin de 1981 :

- 2 paires de surbottes plastique ;

- 2 combinaisons coton ;

- 1 paire de bottes caoutchouc ;

- 3 paires de surbottes plastique à semelles anti-dérapantes :

- 1 paire de gants de coton ;

- 2 paires de gants de chirurgien ;

- 1 paire de gants plastique ; 
- 1 calot ;

- 2 cagoules ;

- 1 tenue plastique ;

- 1 masque respiratoire relié à un ensemble comprenant une alimentation autonome sur batterie, un petit ventilateur, 2 cartouches filtrantes ;

- 1 dispositif de prélèvement d’aérosols avec un ventilateur identique au précédent donc assurant le même débit à travers un filtre papier ;

- 1 détecteur TLD sur le majeur de chaque main ;

- 1 film dosimètre sur chaque poignet ;

- 1 film dosimètre $15 \mathrm{~cm}$ au dessus du genou droit ;

- 1 film poitrine ;

- 2 stylos-dosimètres poitrine (0-1 rem et 0-5 rem, 0-10 mSv et 0-50 mSv) ;

- 1 stylo-dosimètre poignet droit (0-5 rem, 0-50 mSv) sur la $2^{e}$ combinaison permettant une lecture directe de la dose a tout moment ;

- 1 dosimètre à affichage lumineux au niveau de la ceinture ;

- 1 équipement radio. 